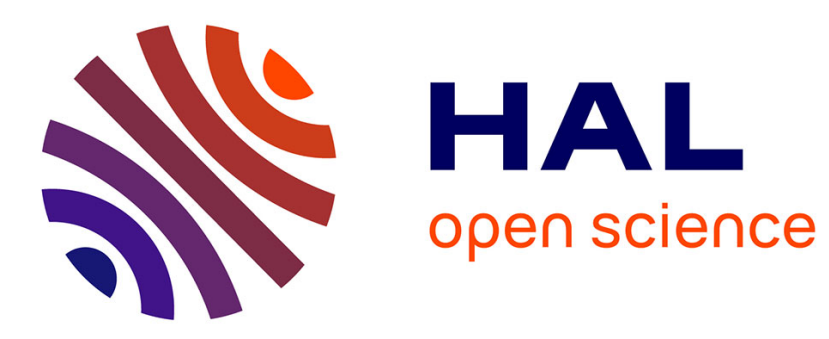

\title{
Le calcul du déplacement chimique, un outil de validation des structures d'acides nucléiques
}

Florent Barbault, Gérard Lancelot

\section{To cite this version:}

Florent Barbault, Gérard Lancelot. Le calcul du déplacement chimique, un outil de validation des structures d'acides nucléiques. Comptes rendus hebdomadaires des séances de l'Académie des sciences. Série B, Sciences physiques, 2001. hal-02164980

\section{HAL Id: hal-02164980 \\ https://hal.science/hal-02164980}

Submitted on 25 Jun 2019

HAL is a multi-disciplinary open access archive for the deposit and dissemination of scientific research documents, whether they are published or not. The documents may come from teaching and research institutions in France or abroad, or from public or private research centers.
L'archive ouverte pluridisciplinaire HAL, est destinée au dépôt et à la diffusion de documents scientifiques de niveau recherche, publiés ou non, émanant des établissements d'enseignement et de recherche français ou étrangers, des laboratoires publics ou privés. 


\title{
Le calcul du déplacement chimique, un outil de validation des structures d'acides nucléiques
}

\author{
Florent Barbault, Gérard Lancelot*
}

Centre de biophysique moléculaire, CNRS, rue Charles-Sadron, 45071 Orléans cedex 2, France

Reçu le 14 mai 2001 ; accepté le 10 septembre 2001

\begin{abstract}
The chemical shift calculation, a tool for nucleic acids structure validation. The large majority of analytical NMR methods in chemistry or biochemistry are based on the quality of the chemical shift dispersion. The purpose of this work is to show that theoretical chemical shift back calculation starting from structures can be used to select molecular modeling structures in order to differentiate several conformational possibilities. Here we report the result of the chemical shift calculation carried out on two original structures corresponding to two DNA 'kissing complexes'. The 46 nucleotides sequence corresponds to the RNA deoxyribose analogous implied in the HIV- $1_{\text {Lai }}$ dimerization process. It is interesting to note that, even in cases we are very far from the classical helical structure (loop-loop interaction, AA base pairing, base stacking, misappariement...), the theoretical chemical shift is in very good agreement with the experimental chemical shift $( \pm 0.25 \mathrm{ppm})$. The satisfactory results obtained enable us to conclude that the comparison of the proton chemical shifts is an invaluable tool making it possible to select or to validate oligonucleotides structures. C 2001 Académie des sciences / Éditions scientifiques et médicales Elsevier SAS
\end{abstract}

NMR / chemical shift calculation / DNA / nucleic acids / kissing complex

Résumé - La grande majorité des analyses de spectres RMN en chimie ou en biochimie repose sur la qualité en dispersion de leur déplacement chimique. L'objectif de ce travail est de montrer que le calcul en retour du déplacement chimique théorique à partir de structures peut être utilisé pour sélectionner des structures issues de la modélisation moléculaire, afin de différencier plusieurs possibilités conformationnelles. Nous présentons ici le résultat du calcul de déplacement chimique effectué sur deux structures originales : deux kissing complexes d'ADN de 46 nucléotides, analogue désoxyribose de la séquence d'ARN impliquée dans le phénomène de dimérisation du virus d'HIV- $1_{\text {Lai }}$. Il est remarquable de constater que, même dans des cas très éloignés de la structure hélicoïdale (interaction boucle-boucle, paire AA, stacking, mésappariement...), le déplacement chimique théorique est en bon accord avec le déplacement chimique expérimental $( \pm 0,25 \mathrm{ppm})$. Les résultats satisfaisants obtenus nous permettent de conclure que la comparaison des déplacements chimiques du proton est un outil précieux permettant de sélectionner ou de valider des structures d'oligonucléotides. (C) 2001 Académie des sciences / Éditions scientifiques et médicales Elsevier SAS

RMN / calcul du déplacement chimique / ADN / acide nucléiques / kissing complexes

Le déplacement chimique est une fonction de la densité électronique présente aux alentours du noyau considéré. Il existe donc une corrélation étroite entre le déplacement chimique et la structure tridimensionnelle d'une macromolécule, bien que l'interprétation structurale du déplacement chimique dans les acides nucléiques soit délicate.
Dans le cas des protéines, il a été montré 11 que le déplacement chimique de l'hydrogène $\alpha$ renseigne directement sur la structure secondaire (hélice $\alpha$, feuillet $\beta$ ) de cette protéine. De plus, dans le logiciel de modélisation moléculaire CNS [2], un potentiel de contrainte utilisant le déplacement chimique du proton $[3,4$ ou du carbone $13[5]$, peut

\footnotetext{
* Correspondance et tirés à part.

Adresse e-mail : lancelot@cnrs-orleans.fr (G. Lancelot).
} 
être utilisé, pour les protéines, au même titre que l'effet nOe ou les constantes de couplages. Quelques essais prometteurs de détermination de l'angle glycosidique à partir du déplacement chimique du ${ }^{13} \mathrm{C}$ ont été mis en ouvre il y a quelques années घ] mais l'application de cette méthode reste très ponctuelle. Il est néanmoins possible de calculer le déplacement chimique théorique à partir d'une structure tridimensionnelle $8-15$.

Il y a quelques années, le logiciel Nuchemics [16], prenant en compte l'équation générale du calcul de déplacement chimique, a été développé. Il a été testé sur des ADN canoniques et de petites "tiges boucles" d'ADN. Il est donc en théorie possible de confirmer une structure hélicoïdale par l'analyse en retour de ses déplacements chimiques, mais son utilisation dans l'analyse de séquences polymorphiques d'ADN mérite d'être testée.

Nous nous sommes intéressés dans ce travail à l'étude d'une séquence de l'ADN dont l'analogue d'ARN a été largement décrit, puisqu'il s'agit de la séquence d'initiation à la dimérisation du virus HIV- $1_{\text {Lai }}$. Le virus HIV comporte la particularité biologique unique de posséder un génome sous forme diploïde 17], dans lequel l'interaction entre les deux copies d'ARN se fait sur une région d'environ 35 nucléotides près de l'extrémité 5', nommé SL1 18-20. Cette séquence comporte deux structures possibles: la première 21] appelée kissing complex, représente un dimère instable, puisqu'il évolue spontanément vers une autre forme, plus stable thermodynamiquement. Ce dimère se compose de deux " tiges boucles " en interaction via l'appariement entre les deux monomères par la séquence palindromique GCGCGC. La deuxième structure [22] correspond au dimère stable de cette séquence d'ARN et représente une structure étendue des deux brins, plus proche d'une forme hélicoïdale classique.

Un des principaux problèmes rencontrés avec ce type de structure réside dans le fait que le spectre proton ne renseigne pas directement sur la nature du dimère. Bien que l'étude d'un échantillon marqué permette de déterminer la forme présente en solution, la discrimination devient délicate en l'absence d'échantillon enrichi et nécessite une double modélisation moléculaire 222. En revanche, le calcul en retour du déplacement chimique permet de sélectionner rapidement les structures valables.

L'échantillon étudié correspond à l'analogue désoxyribose de la séquence SL1, soit l'oligonucléotide d(CTTGCTGAAGCGCGCACGGCAAG) 2 . Comme dans le cas de l'ARN, une courbe de fusion permet de mettre en évidence deux types de dimères, correspondant à deux méthodes de préparations différentes. L'obtention des dimères stable et instable se fait en chauffant l'échantillon à $90^{\circ} \mathrm{C}$ pendant $4 \mathrm{~min}$, puis en le refroidissant lentement ou brusquement à $4{ }^{\circ} \mathrm{C}$, respectivement. Les deux structures ont été déterminée par RMN et sont en cours de publication.

Le dimère instable est une structure de type kissing complex comportant une interaction boucleboucle de quatre paires de bases (figure . Un des éléments structuraux intéressants est la formation d'une paire de base adénine-adénine inter-brin entre le résidu A8 d'un brin et le résidu A8 de l'autre brin. De plus, la base C15 est intercalée entre le résidu G14 et le résidu A9 de l'autre brin. Ces éléments structuraux sont très bien définis par les données RMN "classiques" (effets nOe et constantes de couplage), mais aussi par le calcul en retour du déplacement chimique. En effet, le calcul

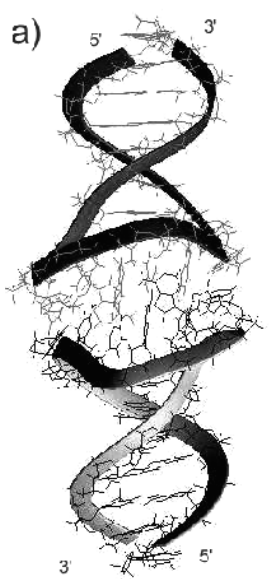

b)

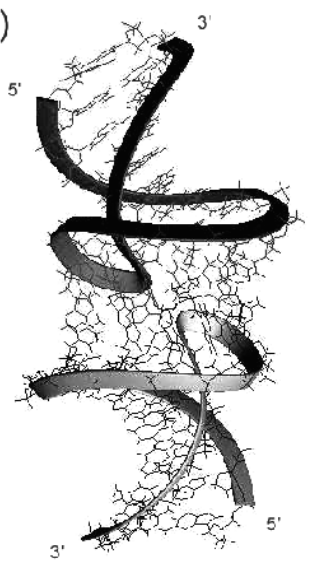

c)

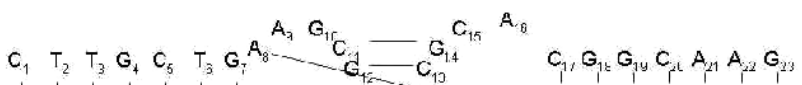

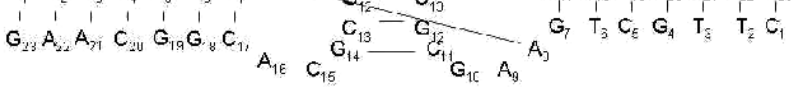

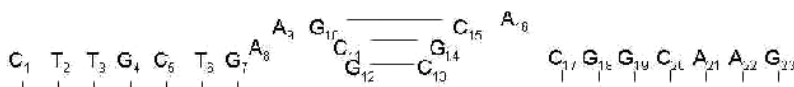

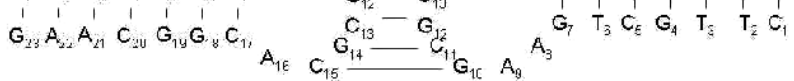

Figure 1. Structure des deux kissing complexes. a. Le dimère instable exhibe une interaction boucle-boucle de quatre paires de bases. b. Le dimère stable présente une interaction boucle-boucle de six paires de base. c. Représentation schématique des deux types de kissing complexes: en haut dimère instable, en bas dimère stable. 
sur les protons non échangeables des 16 structures qui ont convergés donne des écarts inférieurs à $0,25 \mathrm{ppm}$. Cette correspondance entre les données expérimentales et les structures issues de la modélisation moléculaire apporte une preuve supplémentaire importante de la véracité de la structure élucidée. De plus, elle permet une interprétation plus fine, car plus sûre, de la position d'un résidu. Elle montre, par exemple, que le motif de stacking n'est pas un artefact dû au logiciel de modélisation, mais est bien présent sur la structure moyenne.

La deuxième structure de cette séquence représente le dimère thermodynamiquement le plus stable. C'est aussi un kissing complex présentant une interaction boucle-boucle plus importante (six paires de bases). L'appariement adénine-adénine disparaît et l'adénine A9 se place en position de stacking avec la paire G10-C15. Il est à noter que le même mode de préparation conduit à un dimère étendu dans le cas de l'ARN [22].

Les spectres RMN des deux espèces présentent évidemment des déplacements chimiques différents. Une des différences $(0,85 \mathrm{ppm})$ concerne le résidu A16, situé en sortie de boucle. Cette différence de déplacement chimique, ainsi que les autres, est corrélée dans les 13 structures issues de la modélisation moléculaire, avec un écart de déplacement chimique ne dépassant pas 0,25 ppm (tableard).

La mise en ouvre du calcul de déplacement chimique est très simple et le calcul est rapide (temps CPU $2 \mathrm{~s}$, espace mémoire 800 ko sur SGI IRIX 6.5 $300 \mathrm{MHz}$ et $128 \mathrm{Mo}$ RAM, pour la molécule étu- diée). Il peut être ainsi utilisé en fin de protocole de modélisation moléculaire pour affiner la sélection des coordonnées. Un exemple de l'apport du calcul de déplacement chimique en retour est représenté sur la figure. La sélection plus drasti

Tableau. Comparaison des déplacements chimiques expérimentaux et théoriques pour le dimère instable et le dimère stable. Aro 1 : proton $\mathrm{H} 8$ ou $\mathrm{H} 6$, Aro 2 : proton $\mathrm{H} 2$ ou $\mathrm{H} 5$ ou $\mathrm{CH} 3$. Les valeurs correspondent à la moyenne, sur l'ensemble des structures, des différentes valeurs calculées et des valeurs expérimentales.

\begin{tabular}{crrrrrr}
\hline & \multicolumn{3}{c}{ Dimère instable } & \multicolumn{3}{c}{ Dimère stable } \\
Nucléotide & Aro 1 & Aro 2 & \multicolumn{1}{c}{ H1' } & Aro 1 & Aro 2 & \multicolumn{1}{c}{ H1 } \\
\hline C1 & $-0,02$ & $-0,05$ & $-0,05$ & $-0,06$ & 0,01 & 0,01 \\
T2 & $-0,01$ & $-0,01$ & $-0,04$ & $-0,03$ & $-0,03$ & $-0,01$ \\
T3 & 0,08 & 0,02 & $-0,08$ & $-0,02$ & $-0,03$ & 0,02 \\
G4 & 0,00 & & $-0,06$ & 0,06 & & 0,01 \\
C5 & $-0,03$ & 0,02 & 0,00 & 0,03 & $-0,04$ & 0,04 \\
T6 & 0,06 & $-0,02$ & $-0,05$ & $-0,02$ & $-0,02$ & $-0,09$ \\
G7 & 0,01 & & $-0,04$ & $-0,04$ & & 0,12 \\
A8 & 0,01 & $-0,01$ & $-0,02$ & 0,00 & $-0,06$ & $-0,03$ \\
A9 & $-0,10$ & $-0,06$ & $-0,03$ & $-0,02$ & $-0,06$ & $-0,08$ \\
G10 & 0,06 & & 0,00 & 0,02 & & $-0,01$ \\
C11 & $-0,05$ & 0,01 & $-0,03$ & $-0,08$ & $-0,02$ & $-0,01$ \\
G12 & $-0,08$ & & 0,01 & 0,07 & & 0,01 \\
C13 & $-0,03$ & 0,01 & $-0,01$ & $-0,05$ & $-0,04$ & 0,03 \\
G14 & $-0,06$ & & 0,07 & $-0,03$ & & $-0,06$ \\
C15 & 0,01 & 0,01 & $-0,03$ & 0,02 & $-0,05$ & 0,00 \\
A16 & $-0,04$ & $-0,03$ & 0,05 & 0,00 & 0,00 & 0,03 \\
C17 & 0,02 & $-0,01$ & 0,00 & 0,10 & 0,09 & 0,00 \\
G18 & 0,00 & & $-0,02$ & $-0,01$ & & 0,02 \\
G19 & 0,04 & & 0,07 & $-0,03$ & & 0,00 \\
C20 & 0,05 & 0,01 & $-0,02$ & 0,00 & 0,01 & $-0,03$ \\
A21 & 0,02 & 0,03 & 0,02 & 0,04 & 0,06 & 0,03 \\
A22 & 0,03 & $-0,04$ & 0,02 & 0,05 & $-0,08$ & $-0,04$ \\
G23 & 0,08 & & 0,08 & $-0,05$ & & 0,00 \\
\hline
\end{tabular}

Figure 2. L'utilisation du déplacement chimique comme méthode supplémentaire de sélection permet d'affiner la précision des résultats. En haut: les 15 structures du dimère stable ont convergé $\left(\mathrm{RMSD}_{\text {global }}=4,55 \pm 1,72 \AA\right.$ et RMSD sur les bases de jonction $=1,71 \pm 0,74 \AA$ ), mais comportent des écarts de déplacement chimique supérieurs à $0,25 \mathrm{ppm}$. Les structures sont donc réintroduites dans le protocole de modélisation moléculaire. En bas, 13 structures sélectionnées par leur écart en déplacement chimique inférieur à $0,25 \mathrm{ppm}$ $\left(\mathrm{RMSD}_{\text {global }}=1,78 \pm 0,80 \AA\right.$ et RMSD sur les bases de jonction $=0,50 \pm 0,19 \AA$ ).
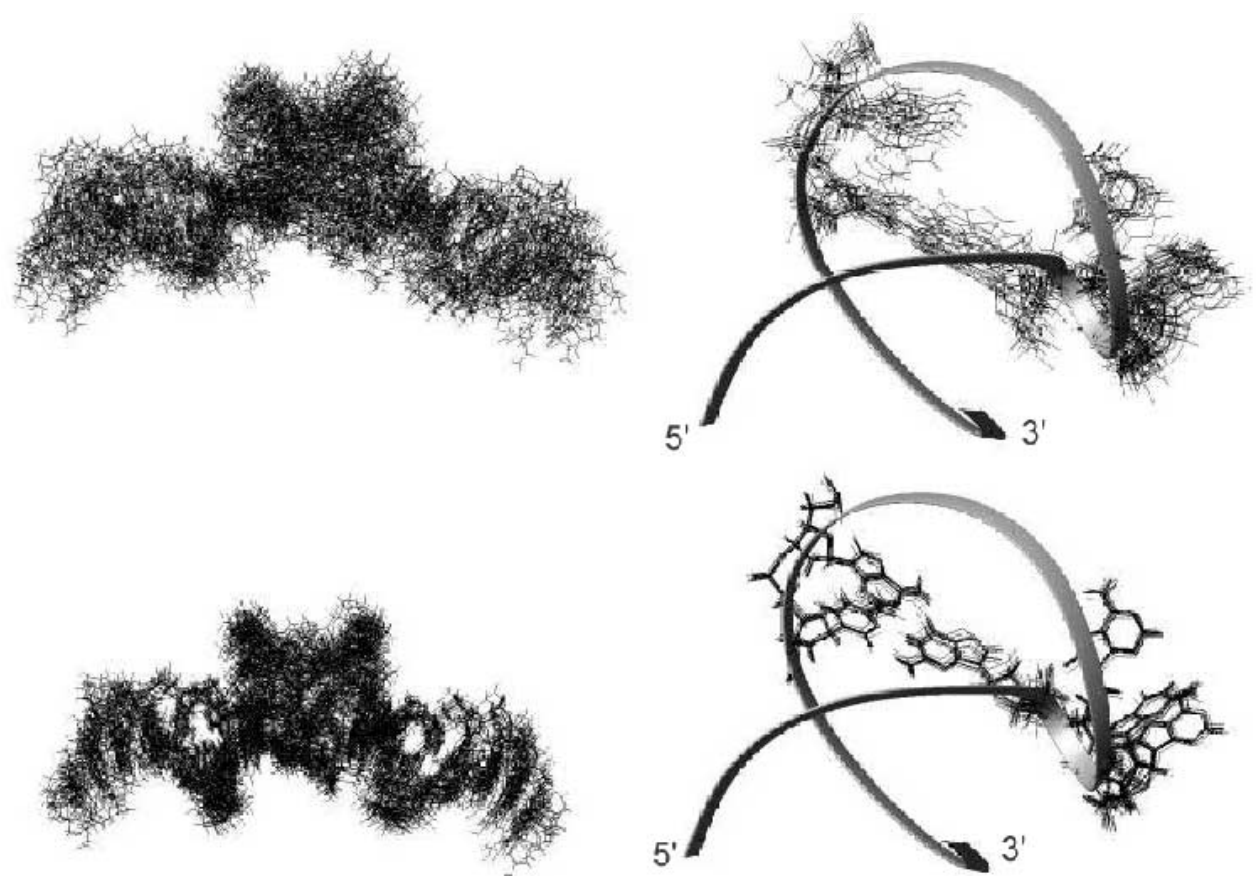
que des coordonnées permet de diminuer le RMSD des régions ayant une forte dynamique et de s'approcher d'une façon plus certaine de la structure moyenne respectant l'ensemble des données RMN

Le déplacement chimique est donc une donnée structurale très riche, dont l'interprétation directe

\section{Références}

[1] Wishart D.S., Sykes B.D., Richards F.M., Biochemistry 31 (1992) 1647.

[2] Brunger A.T., Adams P.D., Clore G.M., DeLano W.L., Gros P., Grosse-Kunstleve R.W., Jiang J.S., Kuszewski J., Nilges M., Pannu N.S., Read R.J., Rice L.M., Simonson T., Warren G.L., Acta Crystallogr. Sect. D 54 (1998) 905.

[3] Kuszewski J., Gronenborn A.M., Clore G.M., J. Magn. Reson. B 107 (1995) 293.

[4] Kuszewski J., Gronenborn A.M., Clore G.M., J. Magn. Reson. B 112 (1996) 79

[5] Kuszewski J., Qin J., Gronenborn A.M., Clore G.M., J. Magn. Reson. B 106 (1995) 92.

[6] Greene K.L., Wang Y., Live D., J. Biomol. NMR 5 (1995) 333.

[7] Ghose R., Marino J.P., Wiberg K.B., Prestegard J.H., J. Am. Chem. Soc. 116 (1994) 8827.

[8] Giessner-Prettre C., Pullman B., Q. Rev. Biophys 20 (1987) 113.

[9] Adamo C., Barone V., Chem. Phys. Lett. 298 (1998) 113.

[10] Case D.A., Curr. Opin. Struct. Biol. 8 (1998) 624.

[11] Case D.A., Curr. Opin. Struct. Biol. 10 (2000) 197. dans le cas des acides nucléiques, sous forme de potentiel de contrainte, est pratiquement impossible à mettre en œuvre. Néanmoins, le calcul en retour sur des structures éloignées des structures "classiques " de l'ADN permet d'obtenir une preuve supplémentaire de la véracité de la structure et apporte une précision notable à la structure moyenne.

[12] Helgaker T., Jaszunski M., Ruud K., Chem. Rev. 99 (1999) 293.

[13] Wilson P.J., Amos R.D., Handy N.C., Mol. Phys. 97 (1999) 757.

[14] De Dios A.C., Roach J.L., Walling A.E., Modelling NMR chemical shifts, Gaining insights into structure and environment, American Chemical Society, Washington DC, 1999, p. 220.

[15] Scheurer C., Skrynnikoc N., Lienin S.F., Straus S.K., Brüschweiler R., Ernst R.R., J. Am. Chem. Soc. 121 (1999) 4230.

[16] Wijmenga S.S., Kruithof M., Hilbers C.W., J. Biomol. NMR 10 (1997) 337

[17] Coffin J.M., RNA tumor virus, Cold Spring Harbor, New York, 1984 , p. 261

[18] Bender W., Davidson N., Cell. 7 (1976) 595.

[19] Kung H.J., Bailey J.M., Davidson N., Nicolson M.O., McAllister R.M., J. Virol. 16 (1975) 397.

[20] Murti K.G., Bondurant M., Tereba A., J. Virol. 37 (1981) 411.

[21] Mujeeb A., Clever J.L., Billeci T.M., James T.L., Parslow T.G., Nat. Struct. Biol. 5 (1998) 432.

[22] Girard F., Barbault F., Gouyette C., Huynh-Dinh T., Paoletti J., Lancelot G., J. Biomol. Struct. Dyn. 16 (1999) 1145.

[23] Takahashi K.I., Baba S., Hayashi Y., Koyanagi Y., Yamamoto N., Takaku H., Kawai G., J. Biochem. 127 (2000) 681. 